\title{
Case report on atypical presentation of Dengue - A patient without fever
}

\section{Rushanthini Seevaamirtham ${ }^{1}$, Chamara Sarathchandra ${ }^{1}$}

Teaching Hospital Anuradhapura ${ }^{l}$.

\begin{abstract}
Dengue can present with wide spectrum of clinical manifestations including atypical presentations. But fever is the most common clinical presentation even with the atypical presentations. Here we report a case of dengue hemorrhagic infection without fever in a 53-year-old female. Clinically and serologically she was diagnosed to have dengue hemorrhagic fever with ultrasound evidence of plasma leakage as well and she was managed successfully and she showed complete recovery without any complications.
\end{abstract}

\section{Introduction}

Dengue is the most important mosquito borne human infection of $21^{\text {st }}$ century. (1) In Sri Lanka the incidence of dengue has markedly increased in last decade and atypical presentations also reported with other co infections. (2) Dengue is a viral infection caused by arbovirus and clinically manifesting with wide range of different presentations from subclinical infection to severe multi organ failure. (3) Even though, fever is the commonest and classic presentation of dengue, here we are reporting a case of dengue infection presented without fever. There are various atypical manifestations of dengue reported so far even though afebrile dengue is a very unusual presentation. Acalculous cholecystitis, acute pancreatitis, myositis, myocarditis, encephalitis, seizures, hepatitis, transverse myelitis, acute respiratory distress syndrome, and renal failure were found as some kind of atypical presentations. $(4,5)$

\section{Case presentation}

53-year-old previously healthy female from Anuradhapura presented with severe headache, nausea and vomiting for one day duration. She did not complain fever. Her systemic inquiry did not reveal any significant symptoms except mild arthralgia and myalgia. Examination revealed temperature of $98.4^{\circ} \mathrm{F}$, blood pressure measured as $120 / 70 \mathrm{mmhg}$, pulse rate was $100 \mathrm{bpm}$ and her neurological examination and other systemic examination was normal. Her headache was persistent and severe until day 9 of the illness and subsided with regular analgesics. Body temperature was normal throughout the course of illness. She does not have any clinical features of immune dysfunction such as recurrent infections.

Her base line full blood count was, WBC- 8600/ $\mu \mathrm{L}, \mathrm{Hb}-11.5$ and platelet 249000. Immediate NCCT brain was taken to rule out possible intracranial pathology and reported as normal. Alumber puncture was performed to rule out possible meningitis and CSF analysis showed clear in appearance and no cells were detected and normal range of proteins. CSF sugar was $84 \mathrm{mg} / \mathrm{dL}$ with RBS of $140 \mathrm{mg} / \mathrm{dL}$. Her ESR was $22 \mathrm{~mm}$ in first hour and CRP was $4.2 \mathrm{mg} / \mathrm{L}$. Her blood, urine and CSF cultures were all negative. Her blood picture revealed reactive lymphocytes suggestive of possible viral infection. Her platelets and white blood cells gradually started to drop and lowest counts were $18000 / \mu \mathrm{L}$ and $1500 / \mu \mathrm{L}$ respectively. Her liver function was monitored and gradually elevated AST and ALT from the base line of $80 \mathrm{u} / 1$ and $40 \mathrm{u} / \mathrm{L}$ to $272 \mathrm{u} / \mathrm{l}$ and $218 \mathrm{u} / \mathrm{L}$ as maximum during the course of illness on day nine and gradually came down from day nine. Ultrasound revealed evidence of plasma leakage on day seven with mild ascites. Her HbA1C was normal and HIV antibodies were negative and possible immune dysfunction is unlikely. 
Her dengue IgM and IgG performed on day six of illness and both were positive and the diagnosis was made as second episode of dengue infection. She was managed with simple analgesics and supportive management with reassurance. Fluid management was given as in dengue pre critical phase and daily bedside ultrasound imaging was performed to detect critical phase early as possible. Vital signs and fluid balance were monitored as per national dengue guidelines. On day seven, she showed ultrasound and clinical evidence of plasma leakage into pleural and peritoneal space and critical phase detected. She was managed as for dengue hemorrhagic fever and critical phase hourly monitoring of parameters started. After day nine of her illness her symptoms gradually subsided and she made complete recovery and discharged from ward on day eleven.

\section{Discussion}

Dengue is a common infectious disease in Sri Lanka even though the atypical presentations of dengue are rare. There are many reported atypical presentations and these can affect any system of the body. The atypical manifestations include quadriplegia, seizure, headache, aseptic meningitis, encephalitis, mononeuropathy and Guillain-Barre. (6) Even though these are atypical presentations, most of these associate with afebrile phase at any time in the course of this illness. Here our patient presented with severe headache as the main complaint, associated with nausea and vomiting without fever.

Cardiac arrhythmias also can occur in the acute phase of dengue fever most likely due to dengue myocarditis and can be an atypical presentation. (7) Sudden onset of severe headache and neurological manifestations might mimic other conditions such as bacterial meningitis. But the blood investigations including elevated hepatic transaminases, thrombocytopenia and leucopenia in a patient in dengue endemic area should raise the suspicion of possibility of dengue even in the absence of a documented fever. To rule out this possibility, serological investigations need to be performed as in our patient.
Dengue meningitis is another atypical rare clinical entity. (8) Here we have excluded the possible meningitis with normal CSF report and inflammatory markers. In a prospective study by Soares et al. from Brazil revealed encephalitis with normal CSF cellularity in $75 \%$ of patients. (8) But dengue presented as meningitis in minority of patients. (9) Dengue virus and dengue IgM isolated from CSF is suggesting that dengue has capacity of causing central nervous system infections as well. (10)

The time of onset of neurological manifestation in a dengue fever is averagely on or after day 10 of the onset of fever. (10) Our patient had severe headache from the first day of illness and does not have any clinical or biochemical picture suggestive of meningitis rather than headache.

Afebrile dengue is an interesting case which is rarely mentioned in the literature. $(11,12)$ The exact pathology of the afebrile dengue in a person without previous comorbidities is not understood yet. (11)A possibility of afebrile dengue is immune suppression such as in long term poorly controlled diabetes mellitus where the inflammatory cytokines (IL-1 and IL-6) secretion by mononuclear cells is suppressed. (12) Consequently there will be no fever in response to infections. Afebrile presentation of dengue in infants also has been reported previously due to immature immune system. (13)

Atypical presentations also end up with critical phase as in our patient and if the diagnosis is missed already, there is a high risk of developing dengue shock and even death without a close precise fluid management and vital parameters monitoring. High clinical suspicion and early recognition of dengue regardless of fever is vital for the successful management especially in dengue endemic areas.

\section{Ethics approval and consent to participate}

Ethical approval was not obtained for the publication of this case report as this does not involve sharing of the personal details of the patient. 


\section{Consent for publication}

Written informed consent was obtained from the patient for publication of this case report.

\section{References:}

1. Dash PK, Parida MM, Saxena P, Abhyankar A, Singh CP, Tewari KN, Jana AM, Sekhar K, Rao PV (2006) Reemergence of dengue virus type-3 (subtype-III) in India: implications for increased incidence of DHF/DSS. Virol J 3: 55.

2. Dandeniya, C. et al., (2015). Atypical manifestations of dengue infection due to co-infection with either hepatitis A or leptospirosis: two case reports. Sri Lankan Journal of Infectious Diseases. 5(1), pp.36-40. (Article).

3. Chamara Dalugama and Indika Bandara Gawarammana, Dengue hemorrhagic fever complicated with acute liver failure: a case report. Journal of Medical Case Reports201711:341. (Article).

4. Ravinder Singh Ahlawat, Tanisha Kalra, Atypical manifestations of dengue fever in a recent dengue outbreak. Annals of Tropical Medicine and Public Health 2017, Volume: 10, Issue : 6, Page : 1448-1452. (Article).

5. Nimmagadda, S. S., Mahabala, C., Boloor, A., Raghuram, P. M., \& Nayak U, A. (2014). Atypical Manifestations of Dengue Fever (DF) - Where Do We Stand Today?. Journal of clinical and diagnostic research : JCDR, 8(1), 71-3.

6. Verma SP, Himanshu D, Tripathi AK, Vaish AK, Jain N. An atypical case of dengue hae- morrhagic fever presenting as quadriparesis due to compressive myelopathy. BMJ Case Rep. 2011; 2011. (Article).

7. Matthias AT, Indrakumar J, Gunatilake SB. Ventricular trigeminy in a patient with serologically confirmed dengue haemorrhagic fever. Int Arch Med. 2014; 7: 28. (Article).

8. Rudra Prosad Goswami1, Arindam Mukherjee1, Tapan Biswas1, Partha Sarathi Karmakar1,2, Alakendu Ghosh3, Two cases of dengue meningitis: a rare first presentation. $J$ Infect Dev Ctries 2012; 6(2):208-211. (Article).

9. Soares CN, Cabral-Castro MJ, Peralta JM, de Freitas MR, Zalis M, Puccioni-Sohler M (2011) Review of the etiologies of viral meningitis and encephalitis in a dengue endemic region. $J$ Neurol Sci 303: 75-79. (Article).

10. VaratharajA (2010) Encephalitis in the clinical spectrum of dengue infection. Neurol India 58: 585-591.(Article)

11. Somsri Wiwanitkit and Viroj Wiwanitkit, Afebrile dengue: An easily forgotten manifestation. Annals of Tropical Medicine and Public Health 2013; Volume 6 Issue 6 Page: 686

12. Wasim Khot and Nitin Gupta et al; A Curious Case of Afebrile Dengue, Journal of The Association of Physicians of India.2018; Vol 66 : 89-90

13. Méndez-Domínguez N, Achach-Medina K, Morales-Gual YM, Gómez-Carro S. Dengue with unusual clinical features in an infant: Case report. Rev Chil Pediatr. 2017 Apr;88(2):275-9. 